Applied Mathematical Sciences, Vol. 9, 2015, no. 10, 481 - 491

HIKARI Ltd, www.m-hikari.com

http://dx.doi.org/10.12988/ams.2015.411893

\title{
An Alternative Definition for the $k$-Riemann-Liouville Fractional Derivative
}

\author{
Gustavo Abel Dorrego \\ Faculty of Exact Sciences \\ National University of Nordeste. Avda. Libertad 5540
}

(3400) Corrientes, Argentina

Copyright (c) 2014 Gustavo Abel Dorrego. This is an open access article distributed under the Creative Commons Attribution License, which permits unrestricted use, distribution, and reproduction in any medium, provided the original work is properly cited.

This work is dedicated to Dr. Rubén A. Cerutti

\begin{abstract}
The aim of this paper is to introduce an alternative definition for the k-Riemann-Liouville fractional derivative given in [6] and whose advantage is that it is the left inverse of the corresponding of k-RiemannLiouville fractional integral operator introduced by [5]. Its basic properties are discussed, their Laplace transform, the derivative of the potential function and the derivative of the Mittag-Leffler k-function introduced in [2] is calculated.
\end{abstract}

Mathematics Subject Classification: 26A33

Keywords: k-Fractional calculus. k-Riemann-Liouville fractional integral

\section{Introduction and Preliminaries}

For further development of this work we need to remember elements of fractional calculus as derivatives and integrals of arbitrary orders. Also remember the action of integral transforms such as Laplace transformation on fractional operators.

Definition 1 Let $f \in L_{\text {loc }}^{1}[a, b]$ where $-\infty \leq a<t<b \leq \infty$. The RiemannLiouville integral of order $\nu$ is defined as 


$$
I^{\nu} f(t):=\frac{1}{\Gamma(\nu)} \int_{a}^{t}(t-\tau)^{\nu-1} f(\tau) d \tau \quad \nu>0
$$

Definition 2 Let $f \in L^{1}[a, b],-\infty \leq a<t<b \leq \infty$ and $I^{n-\nu} f(t) \in$ $W^{n, 1}[a, b], n=[\nu]+1, \nu>0$

The Riemann-Liouville derivative of order $\nu$, is given by

$$
D^{\nu} f(t):=\left(\frac{d}{d t}\right)^{n} I^{n-\nu} f(t)
$$

where $W^{n, 1}[a, b]=\left\{f \in L^{1}[a, b]: f^{(n)} \in L^{1}[a, b]\right\}$ is the Sobolev space.

Definition 3 Let $f: \mathbb{R}^{+} \rightarrow \mathbb{R}$ an exponential order and piecewise continuous function, then the Laplace transform of $f$ is

$$
\mathcal{L}\{f(t)\}(s):=\int_{0}^{\infty} e^{-s t} f(t) d t .
$$

The integral exist for $\operatorname{Re}(s)>0$.

In 2012 Mubeen-Habbibulah (cf.[5]) introduced k-Riemann-Liouville fractional integral given by

Definition 4 Let $\alpha \in \mathbb{R}^{+}$and $n \in \mathbb{N}$ such that $n-1<\alpha<n, f \in L^{1}([0, \infty))$ then the k-Riemann-Liouville fractional integral of $f$ is

$$
I_{k}^{\alpha} f(t)=\frac{1}{k \Gamma_{k}(\alpha)} \int_{0}^{t}(t-\tau)^{\frac{\alpha}{k}-1} f(\tau) d \tau=\frac{t^{\frac{\alpha}{k}-1}}{k \Gamma_{k}(\alpha)} * f(t)
$$

where

$$
\Gamma_{k}(\alpha)=\int_{0}^{\infty} t^{\alpha-1} e^{-\frac{t^{k}}{k}} d t, \quad k>0 .
$$

is the k-Gamma function introduced in [1] and whose relationship with the classical Gamma function is

$$
\Gamma_{k}(\alpha)=k^{\frac{\alpha}{k}-1} \Gamma\left(\frac{\alpha}{k}\right)
$$

Is important to note that for $k \rightarrow 1, \Gamma_{k}(\alpha) \rightarrow \Gamma(\alpha)$, thus $I_{k}^{\alpha} \rightarrow I^{\alpha}$ for $k \rightarrow 1$.

The k-integral (I.4) also satisfies the semigroup property

Proposition 1 Let $\alpha, \beta \in \mathbb{R}^{+}, f \in L^{1}([0, \infty))$ and $k>0$, then

$$
I_{k}^{\alpha} I_{k}^{\beta} f(t)=I_{k}^{\alpha+\beta} f(t)=I_{k}^{\beta} I_{k}^{\alpha} f(t)
$$

Cf.[5] formula (10) p. 91 . 
Proposition 2 Let $f$ be a sufficiently well-behaved function and let $\alpha$ be a real number, $0<\alpha \leq 1$. The Laplace transform of the $k$-Riemann-Liouville fractional integral of the function $f$ is given by

$$
\mathcal{L}\left\{I_{k}^{\alpha} f(t)\right\}(s)=\frac{\mathcal{L}\{f(t)\}(s)}{(k s)^{\frac{\alpha}{k}}}, c f .[6] .
$$

In 2013 [6] introduced the following

Definition 5 Let $\beta \in \mathbb{R}$ such that $0<\beta<1 ; f \in L^{1}([0, \infty))$ and $k>0$ the $k$-Riemnnan-Liouville fractional derivative of order $\beta$ of $f$ is

$$
D_{k}^{\beta} f(t)=\frac{d}{d t} I_{k}^{1-\beta} f(t)
$$

Unfortunately, this derivative is not a left inverse of the corresponding integral operator of the same order as can be verified easily, since

$$
\begin{gathered}
D_{k}^{\alpha}\left(I_{k}^{\alpha} f(t)\right)=\frac{d}{d t}\left[I_{k}^{1-\alpha} I_{k}^{\alpha} f(t)\right] \\
=\frac{d}{d t} I_{k}^{1} f(t)=\frac{d}{d t}\left(\frac{t^{\frac{1}{k}-1}}{k \Gamma_{k}(1)} * f(t)\right) \\
=\frac{d}{d t}\left(\frac{t^{\frac{1}{k}-1}}{k \Gamma_{k}(1)}\right) * f(t) \\
=\frac{1}{k} I_{k}^{1-k} f(t) \neq f(t)
\end{gathered}
$$

This fact led to an alternative definition according to the development of the classical fractional calculus.

Suppose we want to solve the following integral equation (which can be considered as a generalization of the Abel equation of the first kind)

$$
I_{k}^{\alpha} f(t)=u(t)
$$

To solve (I.10), we seek a left inverse operator of the k-Riemann-Liouville fractional integral of the order $\alpha$. To do this we note that, using equation (I.6), we have that the k-Riemann-Liouville fractional integral introduced in [5] it related to the classical Riemann-Liouville fractional integral by

$$
I_{k}^{\alpha} f(t)=k^{-\frac{\alpha}{k}} I^{\frac{\alpha}{k}} f(t)
$$

Therefore, by replacing (I.11) in (I.10) we have 


$$
I^{\frac{\alpha}{k}} f(t)=k^{\frac{\alpha}{k}} u(t)
$$

As $D^{\frac{\alpha}{k}}$ is the inverse operator to the left of $I^{\frac{\alpha}{k}}$ result

$$
f(t)=D^{\frac{\alpha}{k}} k^{\frac{\alpha}{k}} u(t)
$$

In terms of the Riemann-Liouville derivative is:

$$
f(t)=k^{\frac{\alpha}{k}}\left(\frac{d}{d t}\right)^{p} I^{p-\frac{\alpha}{k}} u(t),
$$

where $p=[\alpha / k]+1$.

If we use here once again (I.11)

$$
\begin{aligned}
f(t) & =k^{\frac{\alpha}{k}}\left(\frac{d}{d t}\right)^{p} I^{\frac{p k-\alpha}{k}} u(t) \\
& =\left(\frac{d}{d t}\right)^{p} k^{\frac{\alpha}{k}} k^{\frac{p k-\alpha}{k}} I_{k}^{p k-\alpha} u(t) \\
& =\left(\frac{d}{d t}\right)^{p} k^{p} I_{k}^{p k-\alpha} u(t)
\end{aligned}
$$

This leads us to define a $\mathrm{k}$ version of the classical Riemann-Liouville fractional derivative in terms of k-Riemann-Liouville fractional integral given in $[5]$.

\section{Main results}

Definition 6 Let $k, \alpha \in \mathbb{R}^{+}$and $n \in \mathbb{N}$ such that $n=\left[\frac{\alpha}{k}\right]+1, f \in L^{1}([0, \infty))$ and $I_{k}^{n k-\alpha} f(t) \in W^{n, 1}[a, \infty)$; the modified $k$-Riemann-Liouville fractional derivative is given by

$$
{ }_{k} \mathfrak{D}_{R L}^{\alpha} f(t)=\left(\frac{d}{d t}\right)^{n} k^{n} I_{k}^{n k-\alpha} f(t)
$$

We note at first that, using (I.6) and (I.7) obtained 


$$
\begin{aligned}
& { }_{k} \mathfrak{D}_{R L}^{\alpha} I_{k}^{\alpha} f(t)=\left(\frac{d}{d t}\right)^{n} k^{n} I_{k}^{n k-\alpha}\left(I_{k}^{\alpha} f(t)\right) \\
& =\left(\frac{d}{d t}\right)^{n} k^{n} I_{k}^{n k} f(t) \\
& =k^{n}\left(\frac{d}{d t}\right)^{n} \frac{1}{k \Gamma_{k}(n k)} \int_{0}^{t}(t-\tau)^{\frac{n k}{k}-1} f(\tau) d \tau \\
& =k^{n}\left(\frac{d}{d t}\right)^{n} \frac{1}{k k^{n-1} \Gamma(n k / k)} \int_{0}^{t}(t-\tau)^{n-1} f(\tau) d \tau \\
& =\left(\frac{d}{d t}\right)^{n} \frac{1}{\Gamma(n)} \int_{0}^{t}(t-\tau)^{n-1} f(\tau) d \tau \\
& =\left(\frac{d}{d t}\right)^{n} I^{n} f(t) \\
& =f(t)
\end{aligned}
$$

This proves that indeed the operator ${ }_{k} \mathfrak{D}_{R L}^{\alpha}$ is a left inverse of their corresponding integral operator $I_{k}^{\alpha}$. Note that the above proof also follows that

$$
\left(\frac{d}{d t}\right)^{n} k^{n} I_{k}^{n k} f(t)=f(t)
$$

As a particular case of $k=1$ we have

$$
{ }_{1} \mathfrak{D}_{R L}^{\alpha} f(t)=D_{R L}^{\alpha} f(t),
$$

where $D_{R L}^{\alpha} f(t)$ is a classic fractional derivative of Riemann-Liouville.

Some basic properties of the modified k-Riemann-Liouville fractional derivative are presented.

Proposition 3 Let $f \in L^{1}([0, \infty)), k, \alpha, \beta \in \mathbb{R}^{+}$and $n, m \in \mathbb{N}$ such that $n=\left[\frac{\alpha}{k}\right]+1$ and $m=\left[\frac{\beta}{k}\right]+1$; then

- If $\beta>\alpha$

$$
{ }_{k} \mathfrak{D}_{R L}^{\alpha} I_{k}^{\beta} f(t)=I_{k}^{\beta-\alpha} f(t)
$$

- If $\beta<\alpha$

$$
{ }_{k} \mathfrak{D}_{R L}^{\alpha} I_{k}^{\beta} f(t)={ }_{k} \mathfrak{D}_{R L}^{\alpha-\beta} f(t)
$$

Proof. To prove (II.10), by the semigroup property (I.7)we have

$$
{ }_{k} \mathfrak{D}_{R L}^{\alpha} I_{k}^{\beta} f(t)={ }_{k} \mathfrak{D}_{R L}^{\alpha}\left(I_{k}^{\alpha} I_{k}^{\beta-\alpha} f(t)\right)
$$




$$
=I_{k}^{\beta-\alpha} f(t)
$$

To prove the case (II.11) by using again the semigroup property and (II.9) we have

$$
\begin{aligned}
{ }_{k} \mathfrak{D}_{R L}^{\alpha} I_{k}^{\beta} f(t) & =k^{n}\left(\frac{d}{d t}\right)^{n} I_{k}^{n k-\alpha}\left(I_{k}^{\beta} f(t)\right) \\
& =k^{n-m}\left(\frac{d}{d t}\right)^{n-m}\left(\frac{d}{d t}\right)^{m} k^{m} I_{k}^{(n-m) k-\alpha}\left(I_{k}^{m k} I_{k}^{\beta} f(t)\right) \\
& =k^{n-m}\left(\frac{d}{d t}\right)^{n-m}\left(\frac{d}{d t}\right)^{m} k^{m} I_{k}^{m k}\left(I_{k}^{(n-m) k-\alpha} I_{k}^{\beta} f(t)\right) \\
& =k^{n-m}\left(\frac{d}{d t}\right)^{n-m} I^{(n-m) k-(\alpha-\beta)} f(t) \\
& ={ }_{k} \mathfrak{D}_{R L}^{\alpha-\beta} f(t)
\end{aligned}
$$

Proposition 4 Let $k, \alpha \in \mathbb{R}^{+}$and $r, n \in \mathbb{N}$ such that $n=\left[\frac{\alpha}{k}\right]+1, f \in$ $L^{1}([0, \infty))$ and $I_{k}^{\alpha} f(t) \in A C^{r-1}([0, \infty))=\left\{f \in C^{r-1}([0, \infty)) / f^{(r-1)}\right.$ is absolutely continous $\}$; then

$$
\left(\frac{d}{d t}\right)^{r} I_{k}^{\alpha} f(t)=\frac{1}{k^{r}} I_{k}^{\alpha-r k} f(t), \quad \text { if } \quad r k \leq \alpha .
$$

$$
\left(\frac{d}{d t}\right)^{r} I_{k}^{\alpha} f(t)=\frac{1}{k^{r}} k^{\mathfrak{D}_{R L}^{r k-\alpha}} f(t), \quad \text { if } r k>\alpha
$$

Proof. To prove (II.12) it is sufficient to derive r-times the integral of order $\alpha$.

To prove (II.13), we start with the second member, we take $p=[r k-\alpha]+1$ and we use (II.9)

$$
\begin{aligned}
\frac{1}{k^{r}} \mathfrak{D}_{R L}^{r k-\alpha} f(t) & =\frac{k^{p}}{k^{r}}\left(\frac{d}{d t}\right)^{p} I_{k}^{p k-r k+\alpha} f(t) \\
& =k^{p-r}\left(\frac{d}{d t}\right)^{p} I_{k}^{(p-r) k} I_{k}^{\alpha} f(t) \\
& =\left(\frac{d}{d t}\right)^{r}\left(\frac{d}{d t}\right)^{p-r} k^{p-r} I_{k}^{(p-r) k} I_{k}^{\alpha} f(t) \\
& =\left(\frac{d}{d t}\right)^{r} I_{k}^{\alpha} f(t)
\end{aligned}
$$


Proposition 5 Let $n \in \mathbb{N}$ and $\alpha \in \mathbb{R}^{+}$such that $n=\left[\frac{\alpha}{k}\right]+1, f \in L^{1}([0, \infty))$ and $I_{k}^{n k-\alpha} \in A C^{n}([0, \infty))$; then

$$
I_{k k}^{\alpha} \mathfrak{D}_{R L}^{\alpha} f(t)=f(t)-\left.\sum_{j=1}^{n} k \mathfrak{D}_{R L}^{\alpha-j k} f(t)\right|_{t=0} \frac{t^{\frac{\alpha}{k}-j}}{k \Gamma_{k}(\alpha-j k+k)}
$$

Proof. Making use of (I.6), (II.13) and the derivation rule for a parametric integral notice that

$$
I_{k k}^{\alpha} \mathfrak{D}_{R L}^{\alpha} f(t)=\frac{d}{d t}[\underbrace{\frac{1}{k \cdot k^{\frac{\alpha}{k}-1} \Gamma\left(\frac{\alpha}{k}+1\right)} \int_{0}^{t}(t-\tau)^{\frac{\alpha}{k}} \mathfrak{D}_{R L}^{\alpha} f(\tau) d \tau}_{(*)}]
$$

By integrating by parts n-fold the expression in brackets and applying (II.1)

$$
\begin{gathered}
(*)=\frac{1}{k \cdot k^{\frac{\alpha}{k}-1} \Gamma\left(\frac{\alpha}{k}-n+1\right)} \int_{0}^{t}(t-\tau)^{\frac{\alpha}{k}-n} k^{n} I_{k}^{n k-\alpha} f(\tau) d \tau \\
-\left.\sum_{j=1}^{n} \frac{t^{\frac{\alpha}{k}-j+1}}{k \cdot k^{\frac{\alpha}{k}-1} \Gamma\left(\frac{\alpha}{k}-j+2\right)}\left(\frac{d}{d t}\right)^{n-j} k^{n} I_{k}^{n k-\alpha} f(\tau)\right|_{\tau=0} \\
=\frac{k}{k \Gamma_{k}(\alpha-n k+k)} \int_{0}^{t}(t-\tau)^{\frac{\alpha-n k+k}{k}-1} I_{k}^{n k-\alpha} f(\tau) d \tau \\
-\left.\sum_{j=1}^{n} k^{n}\left(\frac{d}{d t}\right)^{n-j} I_{k}^{n k-\alpha} f(\tau)\right|_{\tau=0} \frac{t^{\frac{\alpha}{k}-j+1}}{k \cdot k^{\frac{\alpha}{k}-1} \Gamma\left(\frac{\alpha-j k}{k}+2\right)} \\
=k I_{k}^{\alpha-n k+k} I_{k}^{n k-\alpha} f(t)-\left.\sum_{j=1}^{n} k^{j-1}{ }_{k} \mathfrak{D}_{R L}^{\alpha-j k} f(\tau)\right|_{\tau=0} ^{\frac{\alpha-j k}{k}+1} \\
=k I_{k}^{k} f(t)-\left.\sum_{j=1}^{n}{ }_{k}^{\frac{\alpha}{k}-1} \mathfrak{D}_{R L}^{\alpha-j k} f(\tau)\right|_{\tau=0} \frac{k^{j-1} t^{\frac{\alpha-j k}{k}+1}}{k^{\frac{\alpha}{k}-1} \Gamma\left(\frac{\alpha-j k}{k}+2\right)}
\end{gathered}
$$

Replacing (II.16) in (II.15) result

$$
\begin{aligned}
I_{k k}^{\alpha} \mathfrak{D}_{R L}^{\alpha} f(t)= & \frac{d}{d t} k I_{k}^{k} f(t)-\left.\sum_{j=1}^{n} k_{k} \mathfrak{D}_{R L}^{\alpha-j k} f(\tau)\right|_{\tau=0} \frac{k^{j-1}}{k^{\frac{\alpha}{k}-1} \Gamma\left(\frac{\alpha-j k}{k}+2\right)}\left(\frac{d}{d t}\right) t^{\frac{\alpha-j k}{k}+1} \\
& =f(t)-\sum_{j=1}^{n} k^{2} \mathfrak{D}_{R L}^{\alpha-j k} f(0) \frac{k^{j-1}}{k^{\frac{\alpha}{k}-1} \Gamma\left(\frac{\alpha-j k}{k}+1\right)} t^{\frac{\alpha}{k}-j}
\end{aligned}
$$




$$
=f(t)-\sum_{j=1}^{n} k_{R L}^{\alpha-j k} f(0) \frac{k^{j-1}}{\Gamma_{k}(\alpha-j k+k)} t^{\frac{\alpha}{k}-j}
$$

Proposition 6 Let $r, n \in \mathbb{N}$ and $\alpha \in \mathbb{R}^{+}$such that $n=\left[\frac{\alpha}{k}\right]+1 ; f \in L^{1}([0, \infty))$ and $I_{k}^{n k-\alpha} f(t) \in A C^{n+r-1}([0, \infty))$, then

$$
\left(\frac{d}{d t}\right)^{r}\left({ }_{k} \mathfrak{D}_{R L}^{\alpha} f(t)\right)=\frac{1}{k^{r}}{ }_{k} \mathfrak{D}_{R L}^{r k+\alpha} f(t)
$$

Proof. From the first member and using (II.13) and (II.1) one has

$$
\begin{aligned}
\left(\frac{d}{d t}\right)^{r}\left({ }_{k} \mathfrak{D}_{R L}^{\alpha} f(t)\right) & =\left(\frac{d}{d t}\right)^{r}\left[\left(\frac{d}{d t}\right)^{n} k^{n} I_{k}^{n k-\alpha} f(t)\right] \\
& =k^{n}\left(\frac{d}{d t}\right)^{n+r} I_{k}^{n k-\alpha} f(t) \\
& =k^{n} \frac{1}{k^{r+n} k} \mathfrak{D}_{R L}^{(n+r) k-n k+\alpha} f(t) \\
& =\frac{1}{k^{r}} \mathfrak{D}_{R L}^{r k+\alpha} f(t)
\end{aligned}
$$

Proposition 7 Let $r, n \in \mathbb{N}$, and $\alpha \in \mathbb{R}^{+}$such that $n=\left[\frac{\alpha}{k}\right]+1$ and $f \in C^{n+r-1}([0, \infty))$; then

$$
{ }_{k} \mathfrak{D}_{R L}^{\alpha} f^{(r)}(t)=\frac{1}{k^{r}} k^{\mathfrak{D}_{R L}^{r k+\alpha}} f(t)-\sum_{j=1}^{r} k^{n-j-1} f^{(r-j)}(0) \frac{t^{-\frac{\alpha}{k}-j}}{\Gamma_{k}(k-j k-\alpha)}
$$

Proof. From (II.1), it result

$$
{ }_{k} \mathfrak{D}_{R L}^{\alpha} f^{(r)}(t)=\left(\frac{d}{d t}\right)^{n} k^{n} I_{k}^{n k-\alpha} f^{(r)}(t)
$$

Calculating the integral $I_{k}^{n k-\alpha} f^{(r)}(t)$ via integration by parts r-times has

$$
\begin{aligned}
& I_{k}^{n k-\alpha} f^{(r)}(t)=\frac{1}{k^{r+1} \Gamma_{k}(n k-\alpha-r k)} \int_{0}^{t}(t-\tau)^{\frac{n k-\alpha-r k}{k}-1} f(\tau) d \tau \\
& \quad-\sum_{j=1}^{r} \frac{f^{(r-j)}(0)}{k^{j+1}} \frac{t^{\frac{n k-\alpha}{k}-j}}{\Gamma_{k}(n k-\alpha-j k+k)} \\
& =\frac{1}{k^{r}} I_{k}^{n k-\alpha-r k} f(t)-\sum_{j=1}^{r} \frac{f^{(r-j)}(0)}{k^{j+1}} \frac{t^{\frac{n k-\alpha}{k}-j}}{\Gamma_{k}(n k-\alpha-j k+k)}
\end{aligned}
$$


Substituting (II.24) into (II.23)

$$
{ }_{k} \mathfrak{D}_{R L}^{\alpha} f^{(r)}(t)=\left(\frac{d}{d t}\right)^{n} k^{n} \frac{1}{k^{r}} I_{k}^{n k-\alpha-r k} f(t)-\sum_{j=1}^{r} \frac{f^{(r-j)}(0)}{k^{j+1}}\left(\frac{d}{d t}\right)^{n} \frac{t^{\frac{n k-\alpha}{k}-j}}{\Gamma_{k}(n k-\alpha-j k+k)}
$$

Applying (II.13) result finally

$$
{ }_{k} \mathfrak{D}_{R L}^{\alpha} f^{(r)}(t)=\frac{1}{k^{r}} k \mathfrak{D}_{R L}^{r k+\alpha} f(t)-\sum_{j=1}^{r} k^{n-j-1} f^{(r-j)}(0) \frac{t^{-\frac{\alpha}{k}-j}}{\Gamma_{k}(k-j k-\alpha)}
$$

Proposition 8 Let $f$ and ${ }_{k} \mathfrak{D}_{R L}^{\alpha} f(t)$ be piecewise continuous and of exponential order, then the Laplace transform of the k-Riemann-Liouville fractional derivative of $f$ is given by

$$
\mathcal{L}\left\{{ }_{k} \mathfrak{D}_{R L}^{\alpha} f(t)\right\}(s)=(k s)^{\frac{\alpha}{k}} \mathcal{L}\{f(t)\}(s)-\sum_{j=0}^{n-1} k(k s)^{j}{ }_{k} \mathfrak{D}_{R L}^{\alpha-j k-k} f(0)
$$

Proof. Applying Laplace transform in the definition (II.1)) and using equations (I.8) and (II.13) is

$$
\begin{gathered}
\mathcal{L}\left\{k_{k} \mathfrak{D}_{R L}^{\alpha} f(t)\right\}(s)=\mathcal{L}\left\{\left(\frac{d}{d t}\right)^{n} k^{n} I_{k}^{n k-\alpha} f(t)\right\}(s) \\
=s^{n} \mathcal{L}\left\{k^{n} I_{k}^{n k-\alpha} f(t)\right\}(s)-\left.\sum_{j=0}^{n-1} s^{j}\left(\frac{d}{d t}\right)^{n-j-1} k^{n} I_{k}^{n k-\alpha} f(t)\right|_{t=0} \\
=k^{n}\left[s^{n} \mathcal{L}\left\{I_{k}^{n k-\alpha} f(t)\right\}(s)-\left.\sum_{j=0}^{n-1} s^{j}\left(\frac{d}{d t}\right)^{n-j-1} I_{k}^{n k-\alpha} f(t)\right|_{t=0}\right] \\
=k^{n}\left[s^{n}(k s)^{-\frac{n k-\alpha}{k}} \mathcal{L}\{f(t)\}(s)-\left.\sum_{j=0}^{n-1} s^{j} k_{k}^{-n+j+1} \mathfrak{D}_{R L}^{\alpha-j k-k} f(t)\right|_{t=0}\right] \\
=(k s)^{n}(k s)^{-\frac{n k-\alpha}{k}} \mathcal{L}\{f(t)\}(s)-\left.\sum_{j=0}^{n-1} s^{j} k_{k}^{j+1} \mathfrak{D}_{R L}^{\alpha-j k-k} f(t)\right|_{t=0} \\
=(k s)^{\frac{\alpha}{k}} \mathcal{L}\{f(t)\}(s)-\left.\sum_{j=0}^{n-1} k(k s)_{k}^{j} \mathfrak{D}_{R L}^{\alpha-j k-k} f(t)\right|_{t=0}
\end{gathered}
$$




\section{Application Examples}

Proposition 9 Let $k, \alpha, \gamma \in \mathbb{R}^{+}$and $n \in \mathbb{N}$ such that $n=\left[\frac{\alpha}{k}\right]+1$ then

$$
{ }_{k} \mathfrak{D}_{R L}^{\alpha}\left(t^{\frac{\gamma}{k}-1}\right)=\frac{k^{n} \Gamma_{k}(\gamma)}{\Gamma_{k}(\gamma-\alpha)} t^{\frac{\gamma-\alpha}{k}-1}
$$

\section{Proof.}

The simple proof is done using definition 6 and formula (11) of [5].

From the above property follows that for $\gamma=k$

$$
{ }_{k} \mathfrak{D}_{R L}^{\alpha}(1)=\frac{k^{n} \Gamma_{k}(k)}{\Gamma_{k}(k-\alpha)} t^{-\frac{\alpha}{k}}=\frac{k^{n}}{\Gamma_{k}(k-\alpha)} t^{-\frac{\alpha}{k}}
$$

That is, the k-Riemann-Liouville derivative of a constant function is not zero unless $\alpha=p k$ for $p \in \mathbb{R}^{+}$.

Proposition 10 Let $\nu, \alpha, \beta, \gamma, k \in \mathbb{R}^{+}$and $n \in \mathbb{N}$ such that $n=\left[\frac{\nu}{k}\right]+1$; then

$$
{ }_{k} \mathfrak{D}_{R L}^{\nu}\left(t^{\frac{\beta}{k}-1} E_{k, \alpha, \beta}^{\gamma}\left(t^{\frac{\alpha}{k}}\right)\right)=k^{n} t^{\frac{\beta-\nu}{k}-1} E_{k, \alpha, \beta-\nu}^{\gamma}\left(t^{\frac{\alpha}{k}}\right)
$$

Where

$$
E_{k, \alpha, \beta}^{\gamma}(z)=\sum_{j=0}^{\infty} \frac{z^{j}(\gamma)_{j, k}}{j ! \Gamma_{k}(\alpha j+\beta)}
$$

is the k-Mittag-Leffler function introduced in [2]

Proof. The simple proof is done using (II.1), the formula (III.1) and the uniform convergence of the serie (III.4).

\section{References}

[1] R. Díaz and E. Pariguan. On hypergeometric functions and k-Pochammer symbol. Divulgaciones Matemáticas Vol.15 2. 2007.

[2] G. Dorrego; R. Cerutti. The k-Mittag-Leffler function. Journal of Applied Math. Int. J. Contemp. Math. Sciences. Vol 7. N¹5. 2012.

[3] R. Hilfer. Threefold introduction to fractional derivatives, Anomalous transport: Foundations and applications. R. Klages, G. Radons and I.M. Sokolov eds. Wiley-VCH Verlong. Weinheim, 2008, pp. 17-73. http://dx.doi.org/10.1002/9783527622979.ch2

[4] A. Kilbas, H. Srivastava and J. Trujillo. Theory and Applications of Fractional Differential Equations. Elsevier. 2006. 
[5] S. Mubeen; G. M. Habibullah. k-Fractional Integrals and Application. Int. J. Contemp. Math, Science. Vol 7 N². 2012.

[6] G. Romero, L. Luque, G. Dorrego, R. Cerutti. On the k-Riemann-Liouville Fractional Derivative. Int. J. Contemp. Math. Science, Vol 8, Nº1, 2013, pp. $41-51$.

Received: November 15, 2014; Published: January 7, 2015 